\title{
| Estudo do diagnóstico de enfermagem fadiga em gestantes atendidas numa unidade básica de atenção à saúde
}

\author{
Study of nursing diagnosis fatigue among pregnant women from a primary care unit \\ Estudio del diagnóstico de enfermería fatiga en gestantes atendidas en una \\ unidad básica de atencion a la salud \\ Viviane Moreira Alves ${ }^{1}$, Zélia Arcanjo Moura', \\ Isaura Letícia Tavares Palmeira', Marcos Venícios de Oliveira Lopes ${ }^{2}$
}

\section{RESUMO}

Objetivo: Analisar o diagnóstico de enfermagem Fadiga em gestantes. Métodos: Estudo transversal com 66 gestantes. Os dados foram coletados através de um roteiro estruturado de entrevista e exame físico. Para a análise foram geradas estatísticas descritivas com a freqüência absoluta e percentual e aplicados testes de associação. Resultados: Do total, 72,7\% apresentaram o diagnóstico de enfermagem Fadiga. As características definidoras mais citadas foram: Aumento das queixas físicas (78,8\%), Sonolência Ambiente barulhento (66,7\%) e Cansaço $(65,2 \%)$, e os fatores relacionados que estiveram mais presentes na amostra foram Estilo de vida enfadonho (54,5\%), Ansiedade (45,5\%) e Anemia (40,9\%). Conclusão: O diagnóstico Fadiga apresenta determinadas características e fatores relacionados importantes, que devem ser foco da atenção por parte dos enfermeiros.

Descritores: Gravidez; Diagnóstico de enfermagem; Saúde da mulher.

\begin{abstract}
Aim: To analyze the nursing diagnosis "fatigue" in pregnant women. Methods: A cross-sectional descriptive design was used to conduct this study. Data were collected from 66 pregnant women using structured interviews and physical examination. Data analysis consisted of descriptive statistics such as frequencies, percentages, and association tests.Results: $72.7 \%$ of pregnant women had fatigue. The most common defining characteristics of fatigue were Increased physical complaints (78.8\%), sleepiness, noisy environmental (66.7\%), and exhaustion $(65.2 \%)$. And, the most common related factors to fatigue were more boring life style $(54.5 \%)$, anxiety (45.5\%), and anemia $(40.9 \%)$. Conclusion: The nursing diagnosis "fatigue" among pregnant women had important defining characteristics and related factors that should be considered and addressed by nurses.

Keywords: Pregnancy; Nursing diagnosis; Woman health.

\section{RESUMEN}

Objetivo: Analizar el diagnóstico de enfermería Fatiga en gestantes. Métodos: Estudio transversal realizado con 66 gestantes. Los datos fueron recolectados a través de una guía estructurada de entrevista y examen físico. Para el análisis se generaron estadísticas descriptivas con la frecuencia absoluta y porcentual y se aplicaron pruebas de asociación. Resultados: Del total, 72,7\% presentaron el diagnóstico de enfermería Fatiga. Las características definidoras más citadas fueron: Aumento de las quejas físicas $(78,8 \%)$, Somnolencia, ambiente bullicioso $(66,7 \%)$ y Cansancio $(65,2 \%)$, y los factores relacionados que estuvieron más presentes en la muestra fueron Estilo de vida aburrido (54,5\%), Ansiedad (45,5\%) y Anemia (40,9\%). Conclusión: El diagnóstico Fatiga presenta determinadas características y factores relacionados importantes, que deben ser foco de atención de los enfermeros.

Descriptores: Embarazo; Diagnósticos de enfermería; Salud de la mujer.
\end{abstract}

\footnotetext{
${ }^{1}$ Enfermeira, integrante do Projeto "Tecnologia para o cuidado de enfermagem" do Departamento de Enfermagem da Universidade Federal do Ceará.

2 Doutor em Enfermagem. Professor Adjunto II do Departamento de Enfermagem da Universidade Federal do Ceará.
} 


\section{INTRODUÇÃO}

A sistematização da assistência de enfermagem é um método organizado e científico que contribui para nortear a prática profissional. Tem como base ações sistemáticas e inter-relacionadas que visam atender às necessidades inerentes de cada indivíduo. Sua característica primordial é a avaliação sistemática dos cuidados de enfermagem.

O processo de enfermagem é o instrumento utilizado na sistematização de enfermagem. Embora comporte variações entre os diversos autores, é constituído por cinco fases: levantamento de dados, diagnóstico de enfermagem, planejamento do cuidado, implementação do cuidado e avaliação dos resultados. O levantamento de dados visa a identificação de sinais e sintomas apresentados pelo indivíduo. Após esta fase, inicia-se o processo diagnóstico no qual os dados coletados são analisados, interpretados, agrupados e nomeados. O planejamento do cuidado determina a assistência de enfermagem a ser prestada ao paciente de acordo com as respostas humanas identificadas. A implementação dos cuidados é a execução do plano de cuidados de enfermagem para suprir as necessidades do paciente. Por fim, a avaliação dos resultados consiste na analise diária das ações implementadas com suas devidas correções ou adaptações ${ }^{(1-2)}$.

$\mathrm{Na}$ fase diagnóstica, que inclui um processo de raciocínio clínico, a nomeação final das respostas humanas identificadas nos clientes é denominada de diagnóstico de enfermagem. O termo diagnóstico de enfermagem teve sua origem na década de 50 , porém, somente na década seguinte, Faye Abdellah apresentou um sistema de classificação para a identificação de 21 problemas de enfermagem. A partir daí, iniciou-se um processo de desenvolvimento de classificações e taxonomias para a enfermagem. Durante os anos 80 ampliou-se a discussão sobre a etapa do diagnóstico de enfermagem, bem como, o interesse por sua aplicação. Os sistemas de classificação existentes foram sendo ampliados, e incorporaram outros termos considerados específicos da prática de enfermagem $^{(3)}$.

Em 2005, foi publicada pela NANDA Internacional a Taxonomia II, constituída de 13 domínios, 46 classes, 167 diagnósticos e sete eixos. Cada domínio é composto por classes, cada classe é composta por diagnósticos. Um eixo tem o propósito de dimensionar e especificar melhor a resposta humana em questão, ou seja, tem o objetivo de compor o enunciado do diagnóstico de enfermagem especificando com fidedignidade as situações clinicas ${ }^{(4-5)}$.

O processo de diagnosticar em enfermagem, como ação independente, pode ser implementado na consulta de enfermagem à gestante. Durante a gestação ocorrem diversas alterações fisiológicas no corpo materno que podem ser identificadas como respostas humanas. Essas alterações ocorrem devido a grande quantidade de hormônios produzidos neste período e ao crescimento fetal. Além das alterações fisiológicas, também ocorrem alterações emocionais e psicológicas que diferem de mulher para mulher..

A maioria dos hormônios que agem durante a gravidez é secretada pela própria placenta. Entre estes hormônios destacam-se o estrogênio e a progesterona, que influenciam as alterações do útero e das mamas que são necessárias para assegurar a vida fetal e promover a produção de leite. Outros dois hormônios importantes são a gonadotropina coriônica e a somatotropina coriônica humana, que têm a função de assegurar a manutenção do concepto até a placenta produzir níveis de progesterona adequados e promover o crescimento fetal ${ }^{(6,7)}$.

$\mathrm{Na}$ Atenção Primária de Saúde uma das portas de entrada das gestantes é o Programa de Saúde da Família (PSF), onde são atendidas gestantes consideradas de baixo risco. $\mathrm{Na}$ consulta de enfermagem, a gestante é questionada quanto a suas queixas e avaliada quanto às alterações físicas durante a realização do exame físico e obstétrico.

Dentre os possíveis diagnósticos de enfermagem apresentados por gestantes, a Fadiga representa uma resposta humana clinicamente importante. Fadiga é uma queixa universal que pode ou não estar relacionada ao diagnóstico médico ou ser resultado de um tratamento ${ }^{(8)}$. Estudiosos de enfermagem têm se interessado pelo estudo da fadiga em diversas situações, inclusive em gestantes. Acredita-se que alterações físicas, como o aumento do volume abdominal, que dificulta o sono, e a dificuldade em dormir, característica do último trimestre da gravidez, possam ser fatores que contribuem para queixas como cansaço freqüente e falta de energia ${ }^{(9-10)}$.

Com base nisso, o presente estudo teve como objetivo analisar o diagnóstico de enfermagem Fadiga apresentado por gestantes acompanhadas numa Unidade Básica de Atenção à Saúde da Família. Especificamente, preocupamo-nos em identificar e verificar associações estatísticas entre as características definidoras, fatores relacionados e o referido diagnóstico.

\section{METÓDOS}

Executiva Regional III, do município de FortalezaCeará. A população estudada foi composta por gestantes acompanhadas pelos enfermeiros da unidade onde o estudo foi desenvolvido. Para a seleção da amostra foram utilizados os dados da população extraídos do Sistema Informatizado de Atenção Básica (SIAB) da unidade, usando o total de gestantes acompanhadas durante o decorrer do ano de 2003. Durante o referido ano foram acompanhadas 269 gestantes representando a população a ser estudada. Para o cálculo da amostra, foi utilizada uma fórmula para estudos de populações pequenas, e 
que leva em consideração o intervalo de confiança, o erro amostral e o tamanho da população ${ }^{(13)}$.

Considerou-se como parâmetro o intervalo de confiança de $95 \%\left(Z_{a}=1,96\right)$ e um erro amostral de $5 \%$ $(\mathrm{E}=0,05)$. A prevalência do fenômeno foi estimada a partir de dados da Secretaria de Saúde do Estado do Ceará, utilizando o total de mulheres em idade fértil em Fortaleza no ano de 2002 (786.764) e o total destas mulheres que buscaram o serviço de obstetrícia no mesmo período (42.430). Com base nesses dados, a prevalência deste fenômeno foi de 5,4\%. Entretanto, considerando a possibilidade de perdas de registro e visando garantir uma maior confiabilidade nos dados, ampliou-se esta prevalência para $6 \%(\mathrm{P}=0,06$ e $\mathrm{Q}=0,94)$. A amostra calculada foi arredondada e estabelecida em 66 indivíduos. O processo de seleção da amostra foi probabilística do tipo randômica simples, a partir de uma tabela de números aleatórios, onde foram incluídas diariamente as gestantes a serem entrevistadas tendo por base a numeração recebida como senha de atendimento ${ }^{(14)}$.

Os critérios de exclusão foram: gestantes sem hemograma; gestantes com qualquer tipo de doença que pudesse interferir no processo de atividade e repouso (doenças cardiovasculares, hematológicas, pulmonares, infecciosas, traumáticas, neurológicas e endócrinas); não aceitar participar do estudo.

$\mathrm{O}$ instrumento utilizado para a coleta dos dados foi um roteiro de entrevista, composto por três partes: dados sócio-econômicos, dados obstétricos e dados referentes a características definidoras e fatores relacionados dos diagnósticos de enfermagem componentes das classes Equilíbrio de energia e Sono e repouso. O instrumento foi desenvolvido com base na taxonomia II da NANDA.

O período de coleta de dados foi de setembro de 2004 a janeiro de 2005 na própria unidade básica, em uma sala específica para a realização do pré-natal, proporcionando um ambiente privativo. Os dados foram coletados através da entrevista com cada gestante e os mesmos foram registrados diretamente no roteiro durante sua aplicação.

Os dados obstétricos foram coletados durante o exame físico da gestante. Para a altura uterina (AU) foi utilizada fita métrica, considerando o espaço entre a sínfise pubiana e o fundo do útero; a idade gestacional foi registrada, em primeira opção, de acordo com os dados da ultrasonografia, em segunda opção pela data da última menstruação (DUM) e, em último caso, pela altura uterina; o peso da gestante foi medido em balança Glicomed mecânica, com piso antiderrapante, capacidade para 120 $\mathrm{kg}$ e precisão que varia em até $3 \%$ do peso real. A balança foi examinada e tarada antes de cada gestante ser avaliada. Os dados de hematócrito e hemoglobina foram extraídos diretamente dos exames laboratoriais realizados pela gestante, e tiveram como principal propósito identificar a presença de anemia na população estudada.

Os dados foram armazenados num banco de dados desenvolvido no Microsoft Excel. Para a análise dos dados foram utilizados os softwares Epi Info for Windows 3.2.2 e o SPSS versão 13.0. A partir dessa análise foram geradas estatísticas descritivas com a freqüência absoluta, percentual, médias, desvio padrão e percentis de cada variável. Em seguida, foram realizadas associações entre algumas variáveis. Os testes aplicados foram o Qui-quadrado e o Teste exato de Fisher. Verificou-se a força da associação através do coeficiente Phi. Calculou-se também a razão de chance. A análise foi baseada na leitura das estatísticas descritivas, bem como na análise do valor $\mathrm{p}$ encontrado, com seus respectivos comentários. Para significância estatística adotou-se um nível de $5 \%(\alpha=0,05)$.

No desenvolvimento do estudo, foram abordados todos os aspectos recomendados na resolução 196/96, sobre pesquisa envolvendo seres humanos ${ }^{(15)}$. A proposta foi encaminhada a Comitê de Ética em Pesquisa para análise e aprovação. Os contatos com a instituição, onde o projeto foi realizado, foram efetivados mediante ofício encaminhado, juntamente com o projeto, à administração local, solicitando autorização para sua realização.

\section{RESULTADOS}

A maioria das gestantes entrevistadas $(59,1 \%)$ tinha de15 a 25 anos de idade, com média de 24 anos $( \pm 5,8)$. Em relação ao estado civil, $78,8 \%$ das gestantes possuíam companheiros. Das gestantes estudadas, $34,8 \%$ apresentaram como grau de escolaridade o ensino fundamental incompleto e 56,1\% trabalhavam como dona de casa. A renda familiar em $86,4 \%$ das entrevistadas variava entre 1 e 3 salários mínimos. Essas mulheres tinham, em média, 4 membros na família $( \pm 2,4)$.

Das gestantes estudadas, a Altura Uterina (AU) média foi de $24,5 \mathrm{~cm}( \pm 7,4)$. Com relação aos exames laboratoriais foram analisados o hematócrito e a hemoglobina para identificar a presença de anemia. A média de hemoglobina foi $11,88 \mathrm{mg} / \mathrm{dl}( \pm 0,97)$. Em relação ao do hematócrito, sua média foi de $35,85 \%$ $( \pm 2,63)$. A Idade Gestacional foi, em média, de 27 semanas. As mulheres estudadas tinham, em média 1 filho $( \pm 1,17)$.

O diagnóstico de enfermagem fadiga, segundo a NANDA (2002), é uma sensação opressiva e sustentada de cansaço e de incapacidade de realizar atividades física e mental habituais. Esse diagnóstico foi encontrado em $72,7 \%$ das gestantes pesquisadas, tendo como principais características definidoras o aumento das queixas físicas, a sonolência, o cansaço, a falta de energia e a incapacidade de restaurar as energias após o sono.

A característica definidora Aumento das queixas físicas diz respeito ao relato da queixa de dor durante a gestação 
e caracterização desta dor quanto à intensidade, freqüência (esporádico, constante e intermitente) e localização segundo o relato das gestantes. A característica sonolência foi selecionada quando a gestante referia constantemente uma vontade de dormir ainda que no período diurno. $\mathrm{O}$ cansaço foi caracterizado pela sensação de exaustão ocasionada pelo mínimo esforço, mesmo que durante o decorrer do dia. A falta de energia estava relacionada ao relato da amostra estudada de não ter forças para realizar tarefas simples ou que comumente não exigiam esforço. A incapacidade de restaurar as energias após o sono foi identificada quando a gestante referia a ocorrência de cansaço ao acordar.

Foram aplicados testes de associação entre as três características definidoras mais freqüentes. Observou-se que as características definidoras não apresentaram associação estatística entre si, e nem com o diagnóstico de enfermagem Fadiga ( $p>0,05)$. Possivelmente devido a uma ocorrência dispersa destas características entre as gestantes, e pelo fato de que gestantes que não apresentaram este diagnóstico (30\% do total) também apresentavam características definidoras isoladas pertencentes ao diagnóstico, mas que não eram suficientes para considerarmos a existência do mesmo.

Tabela 1 - Distribuição das características identificadas na amostra. Fortaleza, 2005.

\begin{tabular}{|c|c|c|c|}
\hline Características definidoras & No. & $\%$ & \\
\hline Aumento das queixas físicas & 52 & 78,8 & \\
\hline Sonolência & 44 & 66,7 & \\
\hline Cansaço & 43 & 65,2 & P75 \\
\hline Falta de energia & 37 & 56,1 & \\
\hline $\begin{array}{l}\text { Incapacidade de restaurar as } \\
\text { energias mesmo após o sono }\end{array}$ & 36 & 54,5 & \\
\hline $\begin{array}{l}\text { Incapacidade de manter o nível } \\
\text { habitual de atividade física }\end{array}$ & 29 & 43,9 & P50 \\
\hline $\begin{array}{l}\text { Aumento das necessidades de } \\
\text { repouso }\end{array}$ & 24 & 36,4 & \\
\hline $\begin{array}{l}\text { Incapacidade de manter as rotinas } \\
\text { habituais }\end{array}$ & 22 & 33,3 & P25 \\
\hline Concentração comprometida & 16 & 24,2 & \\
\hline $\begin{array}{l}\text { Desinteresse quanto ao ambiente } \\
\text { que a cerca }\end{array}$ & 11 & 16,7 & \\
\hline
\end{tabular}

Os fatores relacionados mais freqüentes foram: a temperatura ambiente, o estilo de vida enfadonho, a ansiedade e a anemia. A temperatura ambiente está relacionada à ocorrência de ambiente quente e desconfortável para o sono da gestante. O estilo de vida enfadonho se caracterizou pelo relato das entrevistadas em perceber sua vida monótona e repetitiva, mencionando o motivo para tal percepção. A ansiedade foi selecionada quando a gestante referia sentir-se ansiosa, justificando essa ocorrência. A anemia foi classificada mediante a existência de exames laboratoriais para analise do hematócrito e da hemoglobina, sendo caracterizado como anemia quando um desses parâmetros estivessem abaixo dos padrões de normalidade.

\section{Tabela 2 - Distribuição dos fatores relacionados na} amostra. Fortaleza, 2005.

\begin{tabular}{lcl}
\hline FATORES RELACIONADOS & No & \% \\
\hline Temperatura ambiente & 39 & 59,1 \\
Estilo de vida enfadonho & 36 & 54,5 P75 \\
Ansiedade & 30 & 45,5 \\
Anemia & 27 & 40,9 P50 \\
Barulho & 25 & 37,9 \\
Eventos negativos na vida & 11 & 16,7 P25 \\
Luzes & 8 & 12,1 \\
\hline
\end{tabular}

As associações realizadas entre os quatro fatores relacionados mais citados não mostraram significância estatística. Entretanto, identificou-se associação estatisticamente significante entre o diagnóstico de enfermagem Fadiga e o fator relacionado anemia. A razão de chances mostrou que gestantes com anemia têm $47 \%$ mais chance de desenvolverem Fadiga.

Com relação ao sono, analisou-se a ocorrência concomitante entre as respostas humanas Fadiga, Privação do sono e Padrão de sono perturbado. A associação entre as respostas humanas Fadiga e a Privação de sono apresentou significância estatística $(p=0,014)$, significando que a ocorrência da Fadiga em gestante pode está relacionada com a presença da Privação de sono nas mesmas. $\mathrm{O}$ valor de Phi $=0,303$ confirma que há uma associação moderada entre as duas variáveis. E por fim, a análise da OR refere que, as gestantes que apresentam privação de sono têm 14\% mais de chance de desenvolver fadiga.

O diagnóstico Fadiga também apresentou associação estatisticamente significante com o Padrão de sono perturbado $(p=0,003)$. Entretanto, o valor de Phi ($0,346)$ apontou uma associação moderada e inversa entre os diagnósticos de enfermagem. A razão de chance revelou que as gestantes com Padrão de sono perturbado possuem cerca de 5\% menos chance desenvolver Fadiga. Esta é possivelmente uma associação espúria influenciada pela ocorrência separada dos diagnósticos Privação de sono e Padrão de sono perturbado. Ou seja, em grande parte das gestantes, foram identificados apenas uma das duas respostas humanas referentes ao sono. A ocorrência concomitante do Padrão de sono perturbado e da Privação de sono foi rara, sendo mais comum a identificação desta última resposta.

\section{DISCUSSÃO}

A faixa etária das gestantes pesquisadas estava entre 
15 e 25 anos de idade. Outro estudo realizado no Ceará, revela que $36,0 \%$ das gestantes avaliadas apresentavam a mesma faixa etária do presente estudo. Dados estatísticos do Ministério da Saúde mostram que 25,03\% gestantes cadastradas no SIAB possuíam a faixa etária de 15 à 20 anos de idade. A ocorrência de uma gravidez nesta faixa etária, é objeto de preocupação em Saúde Pública, pois uma gravidez nesta fase da vida é considerada de alto risco devido à ocorrência de um maior número de complicações. Vale ressaltar que, no Ceará, a taxa de gravidez na adolescência vem caindo a cada ano. Em 1997 essa taxa era de 49,3 e em 2002 ela decaiu para 40,4 $4^{(16-18)}$.

O grau de escolaridade das gestantes que participaram da pesquisa é considerado baixo, pois $34,8 \%$ da amostra possuía apenas o ensino fundamental incompleto. Segundo dados estatísticos divulgados no Brasil, a média de anos de estudo em mulheres da região Nordeste é de cinco anos. Outro estudo mostrou que $70 \%$ das gestantes do Ceará possuíam baixa escolaridade ${ }^{(17,19)}$.

Segundo o IBGE ${ }^{(17,19)}$, na região Nordeste $47,5 \%$ das famílias tem uma renda familiar de até dois salários mínimos. No Brasil, a renda familiar em $32,2 \%$ das famílias fica em torno de dois a cinco salários mínimos. No presente estudo $86,4 \%$ das famílias pesquisadas possuíam uma renda familiar de um a três salários mínimos. Outro estudo identificou uma realidade ainda pior, em que $90 \%$ da amostra estudada tinha uma renda menor que um salário mínimo.

As famílias residentes na região Nordeste possuem, em média, quatro pessoas por família ${ }^{(19)}$. Concordando com os dados encontrados em nosso estudo, que mostraram uma média de 4 membros por família.

De acordo com estudo ${ }^{(17)}$ realizado no Ceará, 76,7\% das gestantes tinham de zero a dois filhos, percentual próximo do encontrado nesta investigação $(86,4 \%)$. Acredita-se que, atualmente, os casais estão optando por constituir uma família com média de dois a três filhos.

Com relação à fadiga, alguns autores ${ }^{(6)}$ acreditam que possa estar associada a progesterona e com a ocorrência da anemia fisiológica da gestação. Outros autores ${ }^{(20-21)}$ referem que a gestante está predisposta à fadiga no último trimestre de gestação, em conseqüência ao aumento de peso e as alterações na postura como também a ocorrência de anemia. No presente estudo, não identificamos relação entre a ocorrência de fadiga e os trimestres de gestação.

A associação identificada entre a anemia e a ocorrência de fadiga em gestantes, é justificada pelo o aumento do volume sangüíneo causando uma diminuição da concentração dos eritrócitos ${ }^{(6)}$. Mesmo com os sintomas característicos da anemia e que muito se assemelham às características definidoras da Fadiga, alguns autores ${ }^{(22)}$ são contrários a suplementação de ferro, pois consideram a anemia um fator fisiológico da gravidez não havendo essa necessidade. É importante notar que o número de complicações em mulheres que não recebem suplemento de ferro é muito alto em relação àquelas que recebem. Além disso, crianças de mães que não receberam ferro durante a gravidez têm uma tendência de desenvolver anemia nos primeiros meses de vidas ${ }^{(21)}$.

$\mathrm{O}$ aumento das queixas físicas em gestantes, identificado no presente estudo, pode estar relacionado com a modificação do centro da gravidade, onde o corpo se inclina para trás, encurvando o segmento lombar da coluna e modificando também o eixo da bacia. Pode também, estar associado à pressão do útero aumentado nas estruturas pélvicas de sustentação, à tensão nos ligamentos redondos e ao relaxamento das articulações da bacia ${ }^{(21)}$.

Segundo estudos realizados, a sonolência pode estar relacionada com a ação da progesterona sobre o sistema nervoso central no início da gravidez. Em nosso estudo, identificamos a presença de sonolência, a qual não apresentou associação com o período gestacional que a mulher se encontrava ${ }^{(6)}$.

Neste artigo, o diagnóstico Fadiga apresentou associação estatística e clinicamente importante com a Privação de sono. Estes dados conduzem à possibilidade de se considerar a Privação de sono como fator relacionado à Fadiga. Entretanto, outros estudos são necessários para aprofundar tal relação e estabelecer um encadeamento causal. É necessário considerar que encontramos um número limitado de artigos que discutem a Fadiga em gestantes. Isto influenciou o aprofundamento da discussão dos dados.

\section{CONCLUSÕES}

Durante a gravidez a mulher passa por diversas mudanças, ocasionadas pela explosão hormonal e física desta fase. Essas mudanças precisam ser compensadas e supridas de forma a preservar o seu bem-estar e manter sua saúde. Nesse sentido, o enfermeiro ocupa um papel importante no acompanhamento das gestantes de baixo e de alto risco, que necessitam de recomendações e cuidados para que a gestação seja conduzida a contento, a fim de prevenir a prematuridade e ou riscos à saúde da mãe e do concepto.

Acerca dos resultados obtidos, a maioria das gestantes incluídas no estudo apresentava idade gestacional de 25 a 41 semanas. A maior parte era jovem, com baixa escolaridade e donas de casa. A renda familiar de mais da metade das gestantes era de até um salário mínimo. Quase $60 \%$ da amostra estava passando pela sua primeira gestação.

As características definidoras mais importantes identificadas foram: aumento das queixas físicas, cansaço e sonolência. E os fatores relacionados mais frequentemente identificados foram: temperatura ambiente elevada, estilo de vida enfadonho e ansiedade. Entre as associações estatísticas analisadas, identificamos 
associação significante entre as variáveis fadiga e anemia e entre as variáveis fadiga e privação de sono.

\section{REFERÊNCIAS}

1. Horta WA. Processo de enfermagem. São Paulo: Editora Pedagógica e Universitária - Editora da Universidade de São Paulo; c1979.

2. Lacava RMVB, Barros SMO. Diagnósticos de enfermagem na assistência às gestantes. Acta Paul Enferm. 2004; 17(1): 9-17.

3. Iyer PW, Taptich BJ, Bernocchi-Losey D. Processo e diagnóstico em enfermagem. Porto Alegre: Artes Médicas; 1993.

4. Braga CG, Cruz DALM. A Taxonomia II proposta pela North American Nursing Diagnosis Association (NANDA). Rev Lat. Am Enferm. 2003; 11(2): 2404.

5. North American Nursing Diagnosis Association. Diagnósticos de enfermagem da NANDA: definições e classificação 2003 - 2004. Porto Alegre: Artmed; 2005.

6. Ziegel E, Cranley MS. Enfermagem obstétrica. 8.ed. Rio de Janeiro: Guanabara; 1985.

7. Fischer B. Alterações fisiológicas durante a gestação [texto na Internet]. 2003. [citado 2005 Maio 5]. Disponível: http://w.saudeemmovimento.com.br/ conteudos/onteudo exibe1.asp?cod noticia $=1266$

8. Tiesinga LJ, Dassen TW, Halfens RJ. Fatigue: a summary of the definitions, dimensions, and indicators. Nurs Diagn. 1996; 7(2):51-62. Review.

9. Fawcett J, York R. Spouses' physical and psychological symptoms during pregnancy and postpartum. Nurs Res. 1986; 35(3):144-8.

10. Gardner DL. Fatigue in postpartum women. Appl Nurs Res. 1991; 4(2):57-62.
11. Rouquayrol MZ, Almeida Filho N, organizadores. Epidemiologia \& saúde. 6a ed. Rio de Janeiro: MEDSI; 2003.

12. Cervo AL, Bervian PA. Metodologia científica. 4.ed. São Paulo: Markron Books Ed; 1996.

13. Rea LM, Parker RA. Metodologia de pesquisa: do planejamento à execução. São Paulo: Pioneira; 2002.

14. Jekel JF, Elmore JG, Katz DL. Epidemiologia, bioestatística e medicina preventiva. Porto Alegre: Artmed Editora; 2002.

15. Brasil. Ministério da Saúde. Conselho Nacional de Saúde. Normas de pesquisa envolvendo seres humanos - Res. CNS 196/96. Bioética. 1996; 4(2 Supl):15-25.

16. Ceará. Secretaria de Saúde. Atenção à saúde do adolescente [texto na Internet]. 2002. [citado 2005 maio 5]. Disponível em: www.saude.ce.gov.br/

17. Moura ERF. Assistência de enfermagem no pré-natal no contexto do Programa Saúde da Família [tese]. Ceará: Faculdade de Farmácia, Odontologia e Enfermagem. Universidade Federal de Fortaleza; 2001.

18. Brasil. Ministério da Saúde. DATASUS. Sistema de Informação da Atenção Básica - SIAB. [texto na Internet]. 2004. [citado 2005 Maio 5]. Disponível: www.saude.gov.br./

19. IBGE. Instituto Brasileiro de Geografia e Estatística. Pesquisa nacional por amostra de domicílio: educação e condições de vida [texto na Internet]. 2000. [citado 2005 Maio 5]. Disponível: www.ibge.gov.br/

20. Branden PS. Enfermagem materno-infantil. 2a ed. Rio de Janeiro: Reichmann \& Affonso Editores; 2000.

21. Rezende J, Montenegro CAB. Obstetrícia fundamental. 7 a ed. Rio de Janeiro: Guanabara Koogan; 1996.

22. Polden M, Mantle J. Fisioterapia em obstetrícia e ginecologia. 2a ed. São Paulo: Santos; 1997. 